\title{
Wave Analysis of Liquid Partially Filling a Rotating Cylinder
}

\author{
(Nonlinear Resonance Curve by Shallow Water Theory)*
}

\author{
Fumitaka YOSHIZUMI** \\ **TOYOTA CENTRAL R\&D LABS., INC.,, \\ 41-1, Aza Yokomichi, Oaza Nagakute, Nagakute-cho, Aichi-gun, Aichi 480-1192, Japan \\ E-mail: fyoshi@mosk.tytlabs.co.jp
}

(The author was with Mitsui Engineering \& Shipbuilding Co., Ltd. when this research was performed.)

\begin{abstract}
Wave motion of a liquid in a rotating, axially symmetric container undergoing a whirl motion is investigated. In the analysis of the present study, gravity is considered to be negligible and the liquid motion is taken as being axially uniform. Assuming that the liquid layer is thin, based on the shallow-water theory, the equations of motion are simplified to a one-dimensional problem along the circumference of the container. The modal coupling equations that include the nonlinear effects of the wave motion are obtained by applying Galerkin's method. Periodical solutions under harmonic whirl motion, which give resonance curves, are determined by applying the harmonic balance method to the modal coupling equations. The wave height and the fluid force acting on the container obtained from the analysis are compared with experimental results reported in previous studies. Good agreement is observed between the results of the present analysis and the experimental results with respect to the wave height profile and the magnitude of the fluid force by which the asynchronous whirl motion of the container is excited.
\end{abstract}

Key words: Free Surface Wave, Nonlinear Dynamics, Resonance Curve, Rotor Partially Filled with Liquid, Shallow-Water Theory, Vibration Coupled with Fluid Motion, Vibration of Rotating Body

\section{Introduction}

A self-excited whirl that is asynchronous with respect to the rotating speed occurs in a rotating hollow cylinder that is partially filled with liquid at a particular bound of the rotating speed above the critical speed. The cause of this phenomenon is the free surface wave on a liquid spun up into the form of a ring along the inside wall of the cylinder. The self-excited whirl occurs when the natural frequency of the wave determined by the rotating speed is close to the natural frequency of the elastically supported cylinder. A number of investigators have investigated this phenomenon. In early stages, a number of linear analyses have been performed to investigate natural modes of the free surface wave and instability in the coupling system of the free surface wave and the rotating cylinder on an elastic support system, assuming infinitesimal disturbances ${ }^{(1)-(6)}$. In the next stage, nonlinearities in the free surface wave have been taken into account in order to predict the amplitude of the asynchronous whirl. Berman, Lundgren, and Cheng ${ }^{(7)}$ derived the modified Korteweg-de Vries equation for the retrograde wave traveling on the thin liquid

Received 22 Apr., 2010 (No. T2-06-1127) Japanese Original : Trans. Jpn. Soc. Mech. Eng., Vol.73, No.729, C (2007) pp.1338-1345 (Received 13 Nov., 2006) [DOI: 10.1299/jsdd.4.792]

Copyright $\odot 2010$ by JSME 
layer in a rotating cylinder, in which the shallow-water theory is applied. They attempted to predict the self-excited whirl amplitude in the experiment by considering the coupling system of the liquid wave and the rotating cylinder on an elastic support. Inoue, Jinnouchi, and Araki ${ }^{(8)}$ applied the theory of hydraulic jump to a large wave motion. Under certain conditions of forced whirl motions, they obtained the profile of the free surface wave and the response curves of the wave height and of the fluid force acting on the rotating cylinder as functions of the rotating speed. They compared the analytical results with the results of their experiments. Jorgensen ${ }^{(9)}$ attempted to predict the stationary whirl amplitude in an experiment by applying the theory of hydraulic jump to the formulations derived by Berman, Lundgren, and Cheng. Kasahara, Kaneko, Oshita, and Ishii ${ }^{(10)}$ and Kasahara, Kaneko, and Ishii ${ }^{(11)}$ performed nonlinear time series analysis based on the shallow-water theory in order to investigate nonlinear behavior in free surface waves. As shown above, several investigations taking into account nonlinearities in the free surface wave have been performed. However, a noticeable lack of agreement exists between the theoretical and experimental results on the self-excited whirl amplitude. In addition, few studies have examined the nonlinear response curve of the free surface wave on a liquid enclosed in a rotating cylinder under a steady whirl motion. One exception is a report by Inoue, Jinnouchi, and Araki ${ }^{(8)}$.

The objective of the present investigation is to present a more accurate prediction of the nonlinear actions of a rotor partially filled with liquid. In view of this objective, nonlinear terms that were neglected in the theory by Berman, Lundgren, and Cheng ${ }^{(7)}$ are considered in the theory of the present study. The final formulation used to express nonlinear actions of the wave results in a form of nonlinear coupling between linear modes, which has been applied to common sloshing problems ${ }^{(12)-(17)}$. This leads to an attempt to obtain nonlinear response curves of the free surface wave height and that of the self-excited whirl amplitude. In the present study, we treat the free surface wave in a forced whirling rotor. In a future study, we will treat the coupling problem based on the findings of the present study. Under various experimental conditions, nonlinear analyses are performed in order to obtain nonlinear wave height profiles and the response curves of the wave height and of the fluid force acting on the rotor. In addition, the validity of the theory of the present study is discussed.

\section{Nomenclatures}

$R:$ Radius of the cylindrical cavity

$h$ : Depth of the liquid (liquid layer thickness)

$h_{0}$ : Mean depth of the liquid

$\eta$ : Wave height $\left(h=h_{0}+\eta\right)$

$x, y$ : Tangential coordinate, radial coordinate

(The coordinate system, $O-x y$, is attached to the rotor)

$u, v$ : Tangential fluid velocity, radial fluid velocity

$v_{f}$ : Radial fluid velocity at the free surface

$\Omega$ : Rotating angular speed of the rotor

$\omega$ : Whirl angular frequency of the rotor

$\sigma: \Omega-\omega$

$t$ : Time

$p: \quad$ Pressure of the liquid

$\rho$ : Density of the liquid

$v$ : Kinematic viscosity of the liquid

$v_{T}$ : Kinematic eddy viscosity

$a_{0}$ : Proportionality constant for kinematic eddy viscosity 
E: Ekman number

$X$ : Whirl amplitude of the rotor

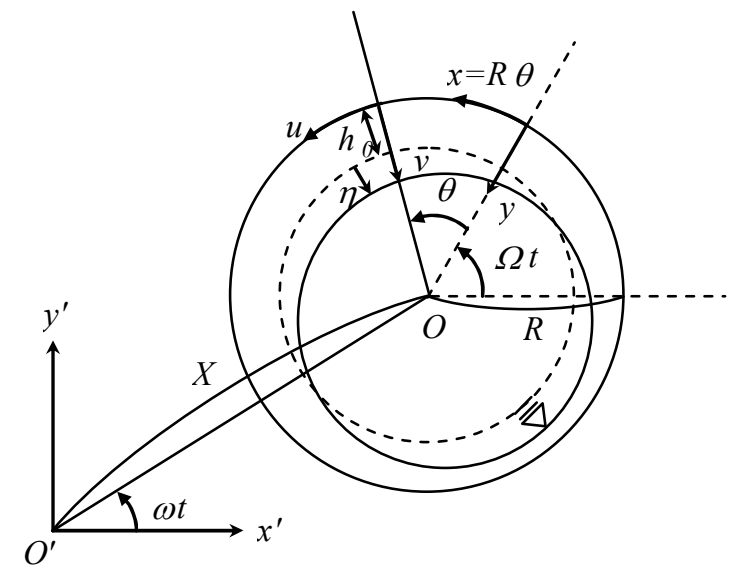

Fig. 1 Model of a whirling rotor partially filled with liquid

\section{Theory}

\subsection{Basic equation and shallow-water approximation}

Figure 1 shows the analytical model used in the present study to consider an axially uniform free surface wave traveling in liquid spun up to a layer along the inside wall of the rotor. The rotor is a rigid cylindrical container that rotates at an angular speed of $\Omega$ and that undergoes a steady whirl motion with an amplitude of $X$ and an angular frequency of $\omega$. For a liquid in the rotor, using a coordinate system attached to the rotor, the equation of motion can be approximately written as follows:

$$
\begin{aligned}
& \frac{\partial u}{\partial t}+u \frac{\partial u}{\partial x}+v \frac{\partial u}{\partial y}-2 \Omega v-\frac{u v}{R}=-\frac{1}{\rho} \frac{\partial p}{\partial x}-X \omega^{2} \sin \left(\sigma t+\frac{x}{R}\right)+v \frac{\partial^{2} u}{\partial y^{2}}, \\
& \frac{\partial v}{\partial t}+u \frac{\partial v}{\partial x}+v \frac{\partial v}{\partial y}+\frac{u^{2}}{R}+2 \Omega u+R \Omega^{2}=-\frac{1}{\rho} \frac{\partial p}{\partial y}-X \omega^{2} \cos \left(\sigma t+\frac{x}{R}\right) .
\end{aligned}
$$

In addition, the equation of continuity is approximately written as:

$$
\frac{\partial u}{\partial x}+\frac{\partial v}{\partial y}=0 .
$$

In the above equations, we assume that the layer is thin compared to the inner radius of the rotor. This assumption allows us to approximate strict equations ${ }^{(4)}$ in such a way as to replace the radius position expressed as $(R-y)$ with the wall position, $R$, and to neglect the viscous term in the radial direction equation of motion, i.e., Eq. (2). This assumption also allows us to neglect the effect of curvature in the equation of continuity, which yields Eq. (3). At the free surface, the kinematic boundary condition and the physical boundary condition are

$$
\begin{array}{ll}
v=v_{f}=\frac{\partial h}{\partial t}+u \frac{\partial h}{\partial x} & (y=h), \\
p=0 & (y=h) .
\end{array}
$$

The boundary condition at the inside wall of the rotor is

$$
v=0 \quad(y=0) \text {. }
$$

Free surface waves on the thin layer can be treated as shallow-water waves. A standard 
approximation of shallow-water theory is to assume that $v$ varies linearly from the inside wall to the free surface, such that

$$
v=\frac{y}{h} v_{f} .
$$

This approximation guarantees the boundary condition of Eq. (6).

In the following, the pressure $p$ is eliminated from equations through a three-step process. First, the pressure $p$ is obtained by integrating Eq. (2) between $y$ and $h$. Second, we differentiate the pressure $p$ obtained in the first step with respect to $x$. Third, we substitute the result of the differentiation into Eq. (1). Before the first step, substituting Eq. (7) into Eq (2) yields

$$
\begin{aligned}
- & \frac{v_{f}}{h^{2}} \frac{\partial h}{\partial t} y+\frac{y}{h} \frac{\partial v_{f}}{\partial t}+\left(\frac{v_{f}}{h}\right)^{2} y+u\left(-\frac{v_{f}}{h^{2}} \frac{\partial h}{\partial x} y+\frac{y}{h} \frac{\partial v_{f}}{\partial x}\right)+\frac{u^{2}}{R}+2 \Omega u+R \Omega^{2} \\
& =-\frac{1}{\rho} \frac{\partial p}{\partial y}-X \omega^{2} \cos \left(\sigma t+\frac{x}{R}\right) .
\end{aligned}
$$

As the first step, integrating Eq. (8) between $y$ and $h$, taking Eq. (5) into account, and substituting Eq. (4) into the result of the integration yields

$$
\frac{p}{\rho}=A+\left\{\frac{u^{2}}{R}+2 \Omega u+R \Omega^{2}+X \omega^{2} \cos \left(\sigma t+\frac{x}{R}\right)\right\}(h-y) .
$$

The specific expression of $A$ is given by Table 1. As the second step, differentiating Eq. (9) with respect to $x$ yields

$$
\begin{aligned}
\frac{1}{\rho} \frac{\partial p}{\partial x}= & \frac{\partial A}{\partial x}+\frac{2 u}{R} \frac{\partial u}{\partial x}(h-y)+\frac{u^{2}}{R} \frac{\partial h}{\partial x}+2 \Omega \frac{\partial u}{\partial x}(h-y)+2 \Omega u \frac{\partial h}{\partial x}+R \Omega^{2} \frac{\partial h}{\partial x} \\
& +\frac{\partial h}{\partial x} X \omega^{2} \cos \left(\sigma t+\frac{x}{R}\right)-\left(\frac{h-y}{R}\right) X \omega^{2} \sin \left(\sigma t+\frac{x}{R}\right) .
\end{aligned}
$$

From Eqs. (3) and (7), we obtain

$$
\frac{\partial u}{\partial x}=-\frac{\partial v}{\partial y}=-\frac{v_{f}}{h} \text {. }
$$

Substituting Eqs. (11) and (4) into Eq. (10) yields

$$
\begin{aligned}
\frac{1}{\rho} \frac{\partial p}{\partial x}= & \frac{\partial A}{\partial x}-\frac{u v_{f}}{R}+\frac{2 u v_{f} y}{R h}-\frac{u}{R} \frac{\partial h}{\partial t}+\frac{2 \Omega v_{f} y}{h}-2 \Omega \frac{\partial h}{\partial t}+R \Omega^{2} \frac{\partial h}{\partial x} \\
& +\frac{\partial h}{\partial x} X \omega^{2} \cos \left(\sigma t+\frac{x}{R}\right)-\left(\frac{h-y}{R}\right) X \omega^{2} \sin \left(\sigma t+\frac{x}{R}\right) .
\end{aligned}
$$

Finally, we substitute Eq. (7) into the left-hand side of Eq. (1) and substitute Eq. (12) into the right-hand side of Eq. (1). Then, integrating the resulting equation between 0 and $h$ and dividing the result by $h$ and substituting Eq. (4) into the resulting equation yields a one-dimensional equation along the circumference, as follows:

$$
\begin{aligned}
\frac{\partial u}{\partial t}+u \frac{\partial u}{\partial x}-2 \Omega \frac{\partial h}{\partial t}+R \Omega^{2} \frac{\partial h}{\partial x}+\frac{1}{3} h \frac{\partial^{3} h}{\partial t^{2} \partial x} \\
=\frac{u}{2 R}\left(3 \frac{\partial h}{\partial t}+u \frac{\partial h}{\partial x}\right)-B-\frac{\partial h}{\partial x} X \omega^{2} \cos \left(\sigma t+\frac{x}{R}\right)-\left(1-\frac{h}{2 R}\right) X \omega^{2} \sin \left(\sigma t+\frac{x}{R}\right) \\
\quad-\left.\frac{v}{h} \frac{\partial u}{\partial y}\right|_{y=0}-\frac{1}{h} \frac{\partial}{\partial x} \int_{0}^{h} \tilde{u}^{2} d y
\end{aligned}
$$

where $B$ represents terms of $(1 / h) \int_{0}^{h}(\partial A / \partial x) d y$ other than $(1 / 3) h\left\{\partial^{3} h /\left(\partial t^{2} \partial x\right)\right\}$, the specific expression of which is given in Table 1 . The velocity $u$ in Eq. (13) means the depth-averaged velocity, while $u$ and $v$ in Eq. (1) are components of the real velocity. In the 
deviation of Eq. (13), under the assumption of shallow-water waves, the velocity $u$ in Eq. (1) is regarded as generally uniform along $y(\partial u / \partial y=0)$ in the domain out of the boundary layer near the wall, and the third term on the left-hand side in Eq. (1) is omitted. There exists turbulence $\widetilde{u}$ that is the difference between the real velocity and the depth-averaged velocity. The turbulence $\tilde{u}$ generates additional stress terms, $\partial \widetilde{u}^{2} / \partial x+\widetilde{u}(\partial v / \partial y)$, which originate from convection terms in Eq. (1), $u(\partial u / \partial x)+v(\partial u / \partial y)\left(=\partial u^{2} / \partial x+\partial(u v) / \partial y \cong \partial u^{2} / \partial x+\right.$ $u(\partial v / \partial y))$. The turbulence develops around bores, and it is expected that $\partial \tilde{u} / \partial x$ is dominant as well as $\partial u / \partial x$ in shallow-water waves. Under the assumption that $\partial \widetilde{u}^{2} / \partial x$ is dominant over $\widetilde{u}(\partial v / \partial y)$ around bores, the term of $(1 / h) \partial\left(\int_{0}^{h} \widetilde{u}^{2} d y\right) / \partial x$ is added as a Reynolds-stress term in Eq. (13). The form proposed by Chester ${ }^{(18)}$ is applied to the wall-friction term, which is the second to last term on the right-hand side of Eq. (13). The Reynolds-stress term, which is the last term on the right-hand side of Eq. (13), is approximated using a spatially constant (but time-dependent) eddy viscosity.

$$
\begin{aligned}
& f=-\left.\frac{v}{h} \frac{\partial u}{\partial y}\right|_{y=0}=-\frac{1}{h} \sqrt{\frac{\nu}{\pi}} \int_{0}^{\infty} \frac{\partial u(t-\tau, x)}{\partial \tau} \frac{d \tau}{\sqrt{\tau}}, \\
& g=-\frac{1}{h} \frac{\partial}{\partial x} \int_{0}^{h} \widetilde{u}^{2} d y=v_{T} \frac{\partial^{2} u}{\partial x^{2}} .
\end{aligned}
$$

These are identical to the procedures proposed by Berman, Lundgren, and Cheng ${ }^{(7)}$. However, in the present procedures, the model ${ }^{(19)}$ such that the constant changes linearly with the wave height and wave velocity, $(R|\sigma|)$, is applied to the eddy viscosity constant. In the present paper, the wave height applied to the constant is the effective value, i.e., the root mean square along the circumference

$$
v_{T}=\sqrt{2} a_{0} R|\sigma| \sqrt{\frac{\int_{0}^{2 \pi R} \eta^{2} d x}{2 \pi R}} .
$$

The equation of continuity is given in the following form:

$$
\frac{\partial u}{\partial x}+\frac{1}{h} \frac{\partial h}{\partial t}=-\frac{u}{h} \frac{\partial h}{\partial x} .
$$

This equation is obtained by substituting the boundary condition at the free surface of Eq. (4) into Eq. (11), which is obtained from Eq. (3).

\subsection{Non-dimensional form}

The lengths are reduced by $h_{0}$, and the time is reduced by $1 / \Omega\left(h_{0}{ }^{\prime}=1, \Omega^{\prime}=1\right)$ to obtain

$$
\left.\begin{array}{l}
R=R^{\prime} h_{0}, \quad h_{0}=h_{0}^{\prime} h_{0}, \quad \eta=\eta^{\prime} h_{0}, \quad \rho=\rho^{\prime} \rho \\
\Omega=\Omega^{\prime} \Omega, \quad u=u^{\prime} h_{0} \Omega, \quad x=R \theta=R^{\prime} h_{0} \theta \\
t=\frac{t^{\prime}}{\Omega}, \quad v=E h_{0}^{2} \Omega, \quad X=X^{\prime} h_{0}, \quad \omega=\omega^{\prime} \Omega
\end{array}\right\} .
$$

In addition, $\sigma^{\prime}=\Omega^{\prime}-\omega^{\prime}$. Introducing the small parameter $\varepsilon$ defined by $X^{\prime}=\varepsilon^{2}$, we set the orders of $u^{\prime}, \eta^{\prime}$ as $u^{\prime}, \eta^{\prime} \sim O(\varepsilon)\left(X^{\prime} \sim O\left(\varepsilon^{2}\right)\right)$, where $O(\varepsilon)$ represents the order of $\varepsilon$. Substituting $h^{\prime}=h_{0}{ }^{\prime}+\eta^{\prime}$ into Eqs. (13) and (17), expanding $1 / h^{\prime}$ in the Taylor expansion as $1 / h^{\prime}=1 / h_{0}{ }^{\prime}-\eta^{\prime} / h_{0}{ }^{2}{ }^{2}+\eta^{\prime 2} / h_{0}{ }^{3}$, and preserving terms of $O(\varepsilon)$ and terms of $O\left(\varepsilon^{2}\right)$ and neglecting terms of $O\left(\varepsilon^{n}\right)(n \geqq 3)$ yields the following equation:

$$
\begin{aligned}
\frac{\partial u}{\partial t} & -2 \Omega \frac{\partial \eta}{\partial t}+\Omega^{2} \frac{\partial \eta}{\partial \theta}+\frac{h_{0}}{3 R} \frac{\partial^{3} \eta}{\partial t^{2} \partial \theta} \\
& =-\frac{1}{R} u \frac{\partial u}{\partial \theta}-\frac{1}{3 R} \eta \frac{\partial^{3} \eta}{\partial t^{2} \partial \theta}+\frac{3}{2 R} u \frac{\partial \eta}{\partial t}-B_{2}-\left(1-\frac{h_{0}}{2 R}\right) X \omega^{2} \sin (\sigma t+\theta)+f+g,
\end{aligned}
$$




$$
\frac{1}{R} \frac{\partial u}{\partial \theta}+\frac{1}{h_{0}} \frac{\partial \eta}{\partial t}=\frac{1}{h_{0}^{2}} \eta \frac{\partial \eta}{\partial t}-\frac{1}{R h_{0}} u \frac{\partial \eta}{\partial \theta},
$$

where $B_{2}$ represents terms of $O\left(\varepsilon^{2}\right)$ in $B$, the specific expression of which is given in Table 1. In the remainder of the present analysis, the symbol ', which indicates a dimensionless quantity, is omitted.

\subsection{Linear natural frequency}

Preserving terms of $O(\varepsilon)$ on the left-hand sides of Eqs. (19) and (20), and eliminating terms on the right-hand sides of Eqs. (19) and (20) yields a linear free problem without disturbances. We assume the solution for the linear free problem to be as follows:

$$
\left.\begin{array}{l}
u=U_{m} \exp \left\{i\left(\sigma_{m} t+m \theta\right)\right\} \\
\eta=H_{m} \exp \left\{i\left(\sigma_{m} t+m \theta\right)\right\}
\end{array}\right\} .
$$

Substituting Eq. (21) into the above-mentioned linear free problem without disturbances yields the eigenvalue problem. Solving this eigenvalue problem, we obtain a pair of natural frequencies $\sigma_{m}$ for each wave number of $m$ as follows:

$$
\sigma_{m}=\frac{\left(m h_{0} / R\right) \Omega}{1+(1 / 3) \cdot\left(m h_{0} / R\right)^{2}}\left[-1 \pm \sqrt{1+\left(R / h_{0}\right)\left\{1+(1 / 3) \cdot\left(m h_{0} / R\right)^{2}\right.}\right\} .
$$

Here, two frequencies are obtained for each $m$. For an observer attached to the rotating system, the equation using the minus sign is for the prograde wave traveling in the same direction as the rotation of the system, and the equation using the plus sign is the retrograde wave traveling in the opposite direction. Natural frequencies under the shallow-water approximation obtained using Eq. (22) are shown in Fig. 2 with strict solutions ${ }^{(1)(6)}$. This figure shows the natural frequencies for $m=1,2$, and 3 with respect to the ratio of depth, $h_{0} / R$. The natural frequencies obtained using the shallow-water approximation are in good agreement with the strict solutions. The natural frequencies are in good agreement even at the range near $h_{0} / R=0.4$. However, since the theory considered in the present paper depends on the shallow-water approximation, we should limit the application of the theory to the small range of $h_{0} / R$ where the tangential velocity in modes can be regarded as uniform along the radial direction.

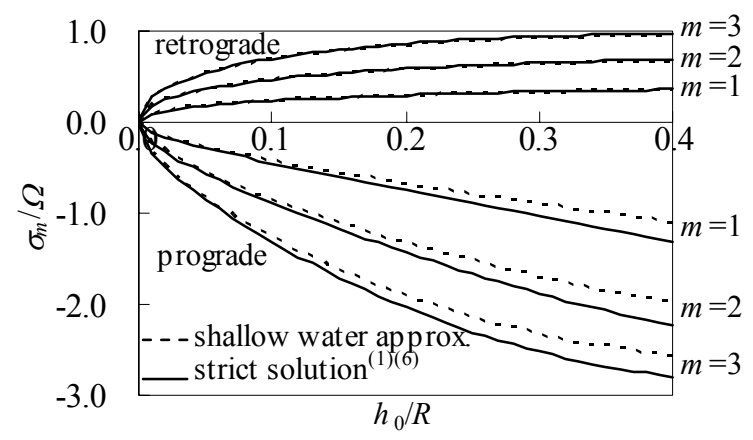

Fig. 2 Natural angular frequencies

\subsection{Nonlinear modal equation}

The nonlinear solution is expressed by the following expansion in linear modes:

$$
\left.\begin{array}{l}
u=\sum_{m}\left\{A_{m}(t) \cos m \theta+B_{m}(t) \sin m \theta\right\} \\
\eta=\sum_{m}\left\{C_{m}(t) \cos m \theta+D_{m}(t) \sin m \theta\right\}
\end{array}\right\} \quad(m \geqq 1) .
$$


The orders of $A_{n}, B_{n}, C_{n}$, and $D_{n}$ are assumed to $O(\varepsilon)$, in the same manner as for $u$ and $\eta$. Substituting Eq. (23) into Eqs. (19) and (20), multiplying the results by $\cos n \theta$ or $\sin n \theta$ and integrating these obtained equations between 0 and $2 \pi$ with respect to $\theta$ results in an equation for each mode. These equations are referred to herein as modal equations. The integration corresponds to the Galerkin method. Applying this operation to Eq. (19) yields

$$
\begin{aligned}
& \dot{B}_{n}-2 \Omega \dot{D}_{n}-n \Omega^{2} C_{n}-\frac{n h_{0}}{3 R} \ddot{C}_{n} \\
& =\frac{1}{2}\left\{\sum_{m=1}^{n-1}\left(K_{1 n-m, m}+K_{2 n-m, m}\right)+\sum_{m=1}^{N-n}\left(K_{1 n+m, m}-K_{2 n+m, m}-K_{1 m, n+m}+K_{2 m, n+m}\right)\right\} \\
& -\delta_{1 n}\left(1-\frac{h_{0}}{2 R}\right) X \omega^{2} \cos \sigma t-F_{r n}\left\{-\operatorname{sign}(\sigma) A_{n}+B_{n}\right\}-n^{2} R_{y} B_{n} \quad(n=1,2, \ldots, N) \\
& \dot{A}_{n}-2 \Omega \dot{C}_{n}+n \Omega^{2} D_{n}+\frac{n h_{0}}{3 R} \ddot{D}_{n} \\
& =\frac{1}{2}\left\{\sum_{m=1}^{n-1}\left(-K_{3 n-m, m}+K_{4 n-m, m}\right)+\sum_{m=1}^{N-n}\left(K_{3 n+m, m}+K_{4 n+m, m}+K_{3 m, n+m}+K_{4 m, n+m}\right)\right\} \\
& -\delta_{1 n}\left(1-\frac{h_{0}}{2 R}\right) X \omega^{2} \sin \sigma t-F_{r n}\left\{\operatorname{sign}(\sigma) B_{n}+A_{n}\right\}-n^{2} R_{y} A_{n} \\
& (n=1,2, \ldots, N) \\
& F_{r n}=\frac{1}{h_{0}} \sqrt{\frac{n E|\sigma|}{2}}, R_{y}=\frac{a_{0}|\sigma|}{R} \sqrt{\sum_{m=1}^{N}\left(C_{m}{ }^{2}+D_{m}{ }^{2}\right)},
\end{aligned}
$$

where $\delta_{m n}$ is the Kronecker delta and the symbol ${ }^{\cdot}$ denotes differentiation with respect to time. As for $F_{r n}$ in Eq. (24), we assume that the amplitude and the phase change slowly and that the whirl motion is regarded as sinusoidal (see Eq. (28)). By substituting Eq. (23), $O(\varepsilon)$-relationships obtained by Eq. (26) $\left(A_{n}=\left(d D_{n} / d t\right) R /\left(n h_{0}\right)\right.$ and $\left.B_{n}=-\left(d C_{n} / d t\right) R /\left(n h_{0}\right)\right)$ and Eq. (28) into Eq. (14), we find the integral on the right-hand side of Eq. (14) expressed by $c_{n}$ and $d_{n}$ yields Fresnel integrals, $\int_{0}^{\infty}(\sin k x / \sqrt{x}) d x=\int_{0}^{\infty}(\cos k x / \sqrt{x}) d x=\sqrt{\pi /(2 k)}(k>0)$. By using these integral identities and using Eq. (28) again to replace $c_{n}$ and $d_{n}$ by $C_{n}$ and $D_{n}$, we get the form of the wall-friction term in Eq. (24) expressed by $F_{r n}$. The number $N$ is the maximum mode order to be taken in the analysis. The specific expressions of $K_{1 m, k} \sim K_{4 m, k}$ are given in Table 1. Applying the same operation to Eq. (20) yields

$$
\begin{aligned}
& -\frac{n}{R} A_{n}+\frac{1}{h_{0}} \dot{D}_{n} \\
& =-\frac{1}{2 h_{0} R}\left[\sum_{m=1}^{n-1} m\left(B_{n-m} D_{m}-A_{n-m} C_{m}\right)\right. \\
& \left.+\sum_{m=1}^{N-n}\left\{m\left(B_{n+m} D_{m}+A_{n+m} C_{m}\right)-(n+m)\left(B_{m} D_{n+m}+A_{m} C_{n+m}\right)\right\}\right] \\
& +\frac{1}{2 h_{0}{ }^{2}}\left\{\sum_{m=1}^{n-1}\left(D_{n-m} \dot{C}_{m}+C_{n-m} \dot{D}_{m}\right)\right. \\
& \left.+\sum_{m=1}^{N-n}\left(D_{n+m} \dot{C}_{m}-C_{n+m} \dot{D}_{m}-D_{m} \dot{C}_{n+m}+C_{m} \dot{D}_{n+m}\right)\right\}(n=1,2, \ldots, N) \\
& \frac{n}{R} B_{n}+\frac{1}{h_{0}} \dot{C}_{n} \\
& =-\frac{1}{2 h_{0} R}\left[\sum_{m=1}^{n-1} m\left(B_{n-m} C_{m}+A_{n-m} D_{m}\right)\right. \\
& \left.+\sum_{m=1}^{N-n}\left\{m\left(-B_{n+m} C_{m}+A_{n+m} D_{m}\right)+(n+m)\left(-B_{m} C_{n+m}+A_{m} D_{n+m}\right)\right\}\right] \\
& +\frac{1}{2 h_{0}^{2}}\left\{\sum_{m=1}^{n-1}\left(-D_{n-m} \dot{D}_{m}+C_{n-m} \dot{C}_{m}\right)\right.
\end{aligned}
$$




$$
\left.+\sum_{m=1}^{N-n}\left(D_{n+m} \dot{D}_{m}+C_{n+m} \dot{C}_{m}+D_{m} \dot{D}_{n+m}+C_{m} \dot{C}_{n+m}\right)\right\}(n=1,2, \ldots, N)
$$

Substituting Eq. (26) into Eq. (24) yields a set of modal equations that is enclosed by $C_{n}$ and $D_{n}$ as shown in Eq. (27), which keep terms of $O(\varepsilon)$ and $O\left(\varepsilon^{2}\right)$ and neglect terms of $O\left(\varepsilon^{n}\right)(n$ $\geqq 3$ ). This substitution process is executed in two steps. In the first step, the only terms of $O(\varepsilon)$ are kept and other terms are neglected to obtain Eq. (26) $\left(A_{n}=\left(d D_{n} / d t\right) R /\left(n h_{0}\right)\right.$ and $B_{n}=$ $-\left(d C_{n} / d t\right) R /\left(n h_{0}\right)$, which is obtained by replacing the right-hand side (nonlinear terms) with zero, is substituted into nonlinear terms in Eq. (26) in order to eliminate $A_{n}$ and $B_{n}$ from these terms. In the second step, the relationship obtained in the first step is substituted into Eq. (24) to eliminate $A_{n}$ and $B_{n}$. In the second step, since terms of $O(\varepsilon)$ and $O\left(\varepsilon^{2}\right)$ are kept and terms of $O\left(\varepsilon^{n}\right)(n \geqq 3)$ are neglected, the case in which only terms of $O(\varepsilon)$ are kept and other terms are neglected in Eq. (26) is sufficient for substitution into the nonlinear terms on the right-hand side of Eq. (24). The specific expressions of $P_{1 m, k} \sim P_{4 m, k}$ and $P_{5 n, m, k} \sim P_{8 n, m, k}$ are given in Table 1.

$$
\begin{aligned}
-2 \Omega \dot{D}_{n} & -n \Omega^{2} C_{n}-\left(\frac{R}{n h_{0}}+\frac{n h_{0}}{3 R}\right) \ddot{C}_{n} \\
= & \frac{1}{2}\left\{\sum_{m=1}^{n-1}\left(P_{1 n-m, m}+P_{2 n-m, m}-P_{5 n, n-m, m}+P_{6 n, n-m, m}\right)\right. \\
& \quad+\sum_{m=1}^{N-n}\left(P_{1 n+m, m}-P_{2 n+m, m}-P_{1 m, n+m}+P_{2 m, n+m}\right. \\
& \left.\left.\quad+P_{5 n, n+m, m}+P_{6 n, n+m, m}+P_{5 n, m, n+m}+P_{6 n, m, n+m}\right)\right\} \\
- & \delta_{1 n}\left(1-\frac{h_{0}}{2 R}\right) X \omega^{2} \cos \sigma t-F_{r n}\left(\frac{R}{n h_{0}}\right)\left\{-\dot{C}_{n}-\operatorname{sign}(\sigma) \dot{D}_{n}\right\}+R_{y}\left(\frac{n R}{h_{0}}\right) \dot{C}_{n}
\end{aligned}
$$

$$
\begin{aligned}
- & 2 \Omega \dot{C}_{n}+n \Omega^{2} D_{n}+\left(\frac{R}{n h_{0}}+\frac{n h_{0}}{3 R}\right) \ddot{D}_{n} \\
= & \frac{1}{2}\left\{\sum_{m=1}^{n-1}\left(-P_{3 n-m, m}+P_{4 n-m, m}+P_{7 n, n-m, m}+P_{8 n, n-m, m}\right)\right. \\
& +\sum_{m=1}^{N-n}\left(P_{3 n+m, m}+P_{4 n+m, m}+P_{3 m, n+m}+P_{4 m, n+m}\right. \\
& \left.\left.\quad+P_{7 n, n+m, m}-P_{8 n, n+m, m}-P_{7 n, m, n+m}+P_{8 n, m, n+m}\right)\right\} \\
- & \delta_{1 n}\left(1-\frac{h_{0}}{2 R}\right) X \omega^{2} \sin \sigma t-F_{r n}\left(\frac{R}{n h_{0}}\right)\left\{-\operatorname{sign}(\sigma) \dot{C}_{n}+\dot{D}_{n}\right\}-R_{y}\left(\frac{n R}{h_{0}}\right) \dot{D}_{n}
\end{aligned}
$$

$$
(n=1,2, \ldots, N)
$$

\subsection{Periodic solution}

We now consider the periodic solution synchronized with the rotating disturbance, the frequency of which is $\sigma$ for an observer attached to the rotor. The solution may be assumed to be in the form

$$
C_{n}=c_{n} \cos n \sigma t+d_{n} \sin n \sigma t, D_{n}=d_{n} \cos n \sigma t-c_{n} \sin n \sigma t \quad(n=1,2, \ldots, N) .
$$

It is also assumed that $c_{n}$ and $d_{n}$ change slowly and that their orders increase by $O(\varepsilon)$ with differentiating once with respect to time. Substituting Eq. (28) into Eq. (27) and maintaining the harmonic balance yields the following equation in terms of $c_{n}$ and $d_{n}$ : 


$$
\begin{aligned}
\left\{I_{2 n}\right. & \left.+\operatorname{sign}(\sigma)\left(\frac{R}{n h_{0}}\right) F_{r n}\right\} \dot{d}_{n}+\left\{\left(\frac{R}{n h_{0}}\right) F_{r n}+\left(\frac{n R}{h_{0}}\right) R_{y}\right\} \dot{c}_{n} \\
= & \left\{I_{1 n}+\operatorname{sign}(\sigma)\left(\frac{R \sigma}{h_{0}}\right) F_{r n}\right\} c_{n}-\left\{\left(\frac{R \sigma}{h_{0}}\right) F_{r n}+\left(\frac{n^{2} R \sigma}{h_{0}}\right) R_{y}\right\} d_{n} \\
& -\frac{\sigma^{2}}{2}\left\{\sum_{m=1}^{n-1}\left(Q_{1 n, n-m, m}+Q_{2 n, n-m, m}\right)\left(c_{n-m} c_{m}-d_{n-m} d_{m}\right)\right. \\
& \left.\quad-\sum_{m=1}^{N-n}\left(Q_{1 n, n+m, m}-Q_{2 n, n+m, m}-Q_{3 n, m, n+m}+Q_{4 n, m, n+m}\right)\left(c_{n+m} c_{m}+d_{n+m} d_{m}\right)\right\} \\
& +\delta_{1 n}\left(1-\frac{h_{0}}{2 R}\right) X \omega^{2} \\
\left\{I_{2 n}\right. & \left.+\operatorname{sign}(\sigma)\left(\frac{R}{n h_{0}}\right) F_{r n}\right\} \dot{c}_{n}-\left\{\left(\frac{R}{n h_{0}}\right) F_{r n}+\left(\frac{n R}{h_{0}}\right) R_{y}\right\} \dot{d}_{n} \\
= & -\left\{I_{1 n}+\operatorname{sign}(\sigma)\left(\frac{R \sigma}{h_{0}}\right) F_{r n}\right\} d_{n}-\left\{\left(\frac{R \sigma}{h_{0}}\right) F_{r n}+\left(\frac{n^{2} R \sigma}{h_{0}}\right) R_{y}\right\} c_{n} \\
& +\frac{\sigma^{2}}{2}\left\{\sum_{m=1}^{n-1}\left(Q_{1 n, n-m, m}+Q_{2 n, n-m, m}\right)\left(c_{n-m} d_{m}+d_{n-m} c_{m}\right)\right. \\
& \left.+\sum_{m=1}^{N-n}\left(Q_{1 n, n+m, m}-Q_{2 n, n+m, m}-Q_{3 n, m, n+m}+Q_{4 n, m, n+m}\right)\left(c_{n+m} d_{m}-d_{n+m} c_{m}\right)\right\}
\end{aligned}
$$

Both equations in Eq. (27) yield the same result. The specific expressions of $I_{1 n}, I_{2 n}$, and $Q_{1 n, m, k} \sim Q_{4 n, m, k}$ are given by Table 1 . We obtain the periodic solution by replacing $\dot{c}_{n}$ and $\dot{d}_{n}$, i.e., the left-hand sides of Eq. (29) with zero.

The stability of the resultant periodic solution can be examined by considering small perturbations with respect to the solution ${ }^{(12)(13)(17)}$. This method is outlined in the following. We define $c_{n}=c_{n 0}+\gamma_{n}$ and $d_{n}=d_{n 0}+\delta_{n}$, where the subscript 0 refers to the obtained periodic solution, which is constant with increasing time, and $\gamma_{n}$ and $\delta_{n}$ are small perturbations with respect to the obtained periodic solution, $\left(c_{n 0}, d_{n 0}\right)$. Substituting $c_{n}=c_{n 0}+$ $\gamma_{n}$ and $d_{n}=d_{n 0}+\delta_{n}$ into Eq. (29), neglecting products of $\gamma_{n}$ and $\delta_{n}$, and considering the fact that $\left(c_{n 0}, d_{n 0}\right)$ is the solution of the simultaneous equation obtained by replacing the left-hand sides of Eq. (29) with zero, the right-hand sides of Eq. (29) also becomes zero, we obtain a set of linear homogeneous first-order differential equations in terms of $\gamma_{n}$ and $\delta_{n}$ with respect to time, i.e., a linear autonomous system. Finally, the eigenvalue problem for the linear system is solved. If all of the real parts of the eigenvalues are negative, the perturbation decreases with increasing time and the periodic solution is stable (realistic), and vice versa.

\subsection{Fluid force}

The pressure force $F_{p}$ and the wall-friction force $F_{r}$ acting on the rotor per unit axial length and the total force $F$, which is obtained as the sum of these forces, are calculated as follows:

$$
\begin{aligned}
& F_{p 1}=\left.R \int_{0}^{2 \pi} p\right|_{y=0} \cos \theta d \theta \\
& F_{p 2}=\left.R \int_{0}^{2 \pi} p\right|_{y=0} \sin \theta d \theta \\
& F_{f r 1}=\rho R \int_{0}^{2 \pi} h f \sin \theta d \theta \\
& F_{f r 2}=-\rho R \int_{0}^{2 \pi} h f \cos \theta d \theta \\
& F_{1}=F_{p 1}+F_{f r 1}, \quad F_{2}=F_{p 2}+F_{f r 2},
\end{aligned}
$$


Table 2 Analytical conditions

\begin{tabular}{|c|c|c|}
\hline condition & (a) & (b) \\
\hline$R[\mathrm{~m}]$ & 0.152 & 0.120 \\
\hline$h_{0}[\mathrm{~m}]$ & \begin{tabular}{l|l:l}
0.010 & 0.020 & 0.040 \\
\end{tabular} & 0.010 \\
\hline$R^{\prime}=R / h_{0}$ & \begin{tabular}{l|l|l}
15.2 & 7.6 & 3.8 \\
\end{tabular} & 12.0 \\
\hline$\omega[\mathrm{rad} / \mathrm{s}]$ & 62.8 & variable \\
\hline$\Omega[\mathrm{rad} / \mathrm{s}]$ & variable & 69.1 \\
\hline$E, \times 10^{-4}$ & $\begin{array}{c}1.59 \quad 0.40 \\
\text { (where } \Omega=\omega)\end{array}$ & 1.45 \\
\hline$X^{\prime}=X / h_{0}{ }^{*}$ & $0 \mid \begin{array}{l}0.025 \\
0.050 \\
0.088\end{array}$ & 0.020 \\
\hline
\end{tabular}

ख: Containers are excited in one direction with the amplitude of $2 \cdot X(4 \cdot X \mathrm{p}-\mathrm{p})$ in all conditions.

$\rho=1000\left[\mathrm{~kg} / \mathrm{m}^{3}\right], v=1.0 \times 10^{-6}\left[\mathrm{~m}^{2} / \mathrm{s}\right]$ in all conditions.

following response curves, solid lines represent stable (realistic) solutions and broken lines represent unstable (unrealistic) solutions.

\subsection{Response of the maximum difference in the wave height profile}

For the case in which $h_{0}=20 \mathrm{~mm}$ and $X=1 \mathrm{~mm}$ in condition (a), the maximum difference $\Delta \eta$ in the wave height profile as a function of $\Omega / \omega$ is shown in Fig. 3. The maximum difference $\Delta \eta$ is the difference between the highest point and the lowest point in the wave height profile. The maximum difference $\Delta \eta$ for the case in which $a_{0}=0.4$ is larger than that for the case in which $a_{0}=1.6$, because, in the case of $a_{0}=0.4$, higher-order modes are encouraged, and the wave height has a profile similar to an undular bore. For both values of $a_{0}$, following the response curve from $\Omega / \omega=1$, we observe the analytical rotating speeds at which the prograde or retrograde wave grows are in good agreement with the experimental values. The response curve of $a_{0}=0.4$ consists of stable solution curves and unstable solution curves. The unstable solution curve indicates that the jump phenomenon occurs by the change in the speed ratio. Remarkable jump phenomena are observed in the experiment by the change in the speed ratio, $\Omega / \omega$. The analysis of $a_{0}=0.4$ also shows the jump phenomenon, although there is a lack of agreement between the rotating speed of the jump phenomenon in the analysis and that in the experiment.
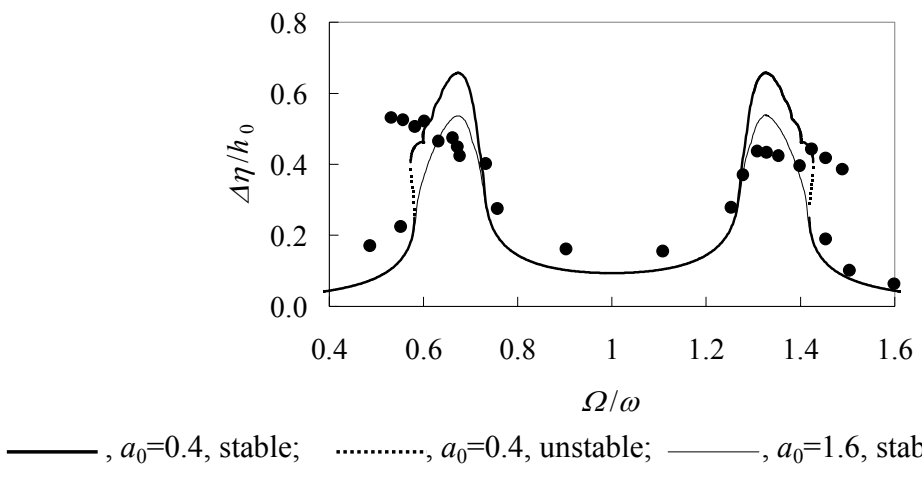

$a_{0}=0.4$, unstable; $a_{0}=1.6$, stable $\ldots . .$. analysis

-..... experiment by Inoue et al. (1985)

Fig. 3 Wave height response (condition (a), $h_{0}=20 \mathrm{~mm}, X^{\prime}=0.050$ )

\subsection{Wave height profile}

Wave height profiles along the circumference are shown in Figs. 4(1) and 4(2) for the case in which $h_{0}=20 \mathrm{~mm}$ and $X=1 \mathrm{~mm}$ in condition (a) and the case of condition (b), respectively. Profiles at the moment when the whirl phase angle coincides with the $\theta=0^{\circ}$ direction are drawn in the figures. Figure 4(1) shows the analytical profiles for the rotating speeds at which the analysis gives the same phase lag of the fluid force as the experiment. The experimental profiles shown in Fig. 4(2) are drawn through two steps. As the first step, 
the reported time series of the wave height is converted to the spatial profile by the rotating speed. As the second step, the $\theta$ position giving the profile its peak is set to that in the analytical profile of $a_{0}=0.4$. In both Figs. 4(1) and 4(2), the analysis using the small value of $a_{0}$ gives a profile similar to an undular bore because of the growth of higher-order modes. As for condition (a) in Fig. 4(1), the experimental wave profile is not oscillatory and the analysis of $a_{0}=1.6$ is in good agreement with the experimental results. On the other hand, for condition (b) in Fig. 4(2), the experimental wave profile is oscillatory, and the analysis of $a_{0}=0.4$ is in good agreement. Note that the analysis of $a_{0}=0.4$ in condition (b) reproduces the number of profile peaks and the change in profile shape caused by a slight change in the rotating speed.

As these experiments show, condition (b) with a depth ratio of $h_{0} / R=0.083\left(R / h_{0}=\right.$ 12.0) gives more oscillatory wave profiles than condition (a) with a depth ratio of $h_{0} / R=$ $0.132\left(R / h_{0}=7.6\right)$. The difference in the wave height profile is predicted to come from the depth ratio $h_{0} / R$. The suitable choice of $a_{0}$ reproduces well the practical wave height profile,
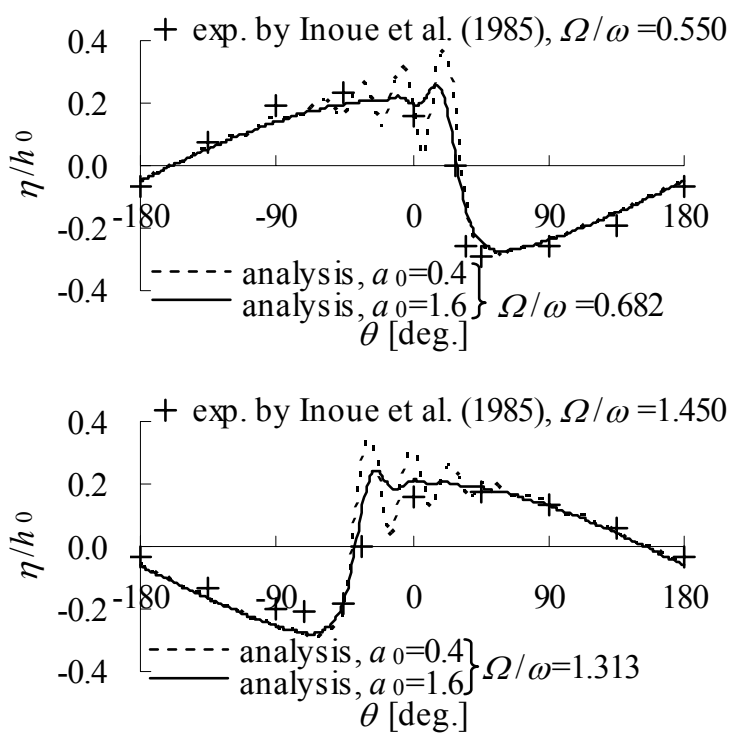

(1) Condition (a), $R / h_{0}=7.6, X^{\prime}=0.050$
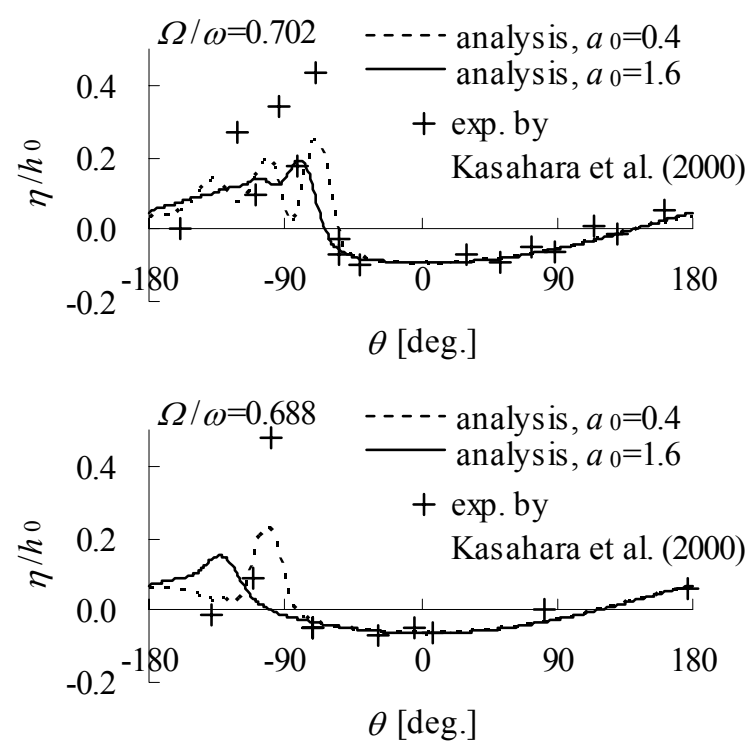

(2) Condition (b), $R / h_{0}=12.0, X^{\prime}=0.020$

Fig. 4 Wave height profile 
as shown in Fig. 4. On the present results of $h_{0} / R=0.083$ and 0.132 , we can predict the tendency that the suitable value of $a_{0}$ increases with increasing $h_{0} / R$, but can not get a more universal relationship between $h_{0} / R$ and the suitable $a_{0}$.

\subsection{Fluid force response}

The fluid force responses for the case in which $h_{0}=20 \mathrm{~mm}$ and $X=1 \mathrm{~mm}$ in condition (a) and for the case of condition (b) are shown in Figs. 5(1) and 5(2), respectively. Here, $F_{R}$ ' and $F_{I}$ ' are the dimensionless fluid forces given by $F_{R}{ }^{\prime}=F_{R} /\left(\rho h_{0}{ }^{3} \Omega^{2}\right)$ and $F_{I}{ }^{\prime}=F_{I} /\left(\rho h_{0}{ }^{3} \Omega^{2}\right)$, respectively, where $F_{R}$ and $F_{I}$ are the real part and the imaginary part of the fluid force acting on the cylindrical container per unit axial length. The imaginary part corresponds to the excitation force in the asynchronous self-excited whirl. Unlike the response curves of the wave height, the response curves of the fluid force for $a_{0}=0.4$ are not smooth (see Fig. 3 ). However, the change in $a_{0}$ results in only a slight change in peak values of the fluid force.
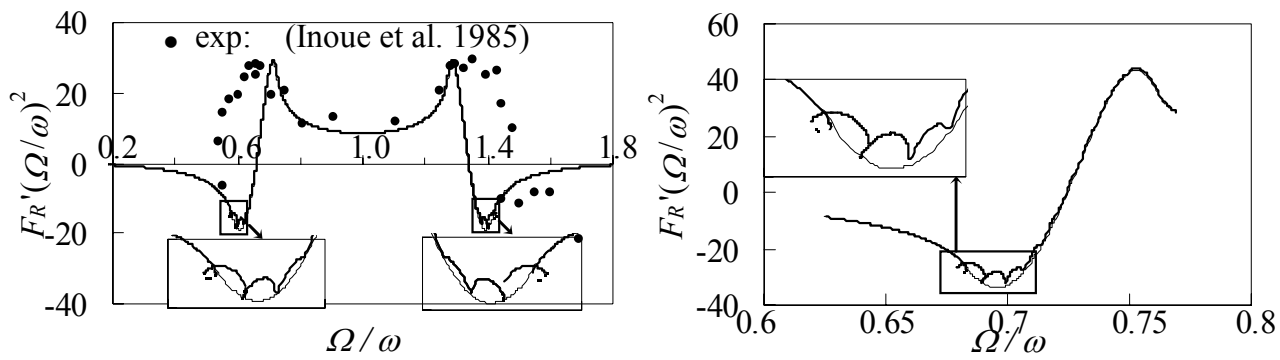

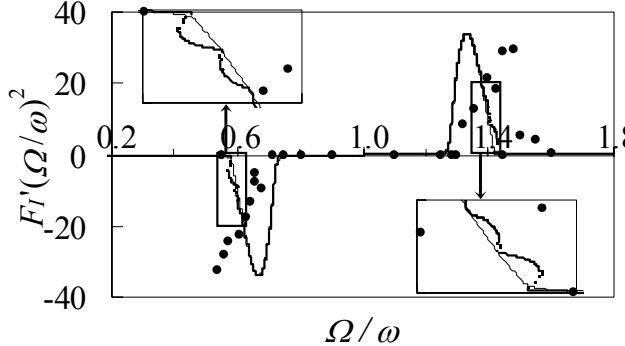

(1) Condition (a), $R / h_{0}=7.6, X^{\prime}=0.050$

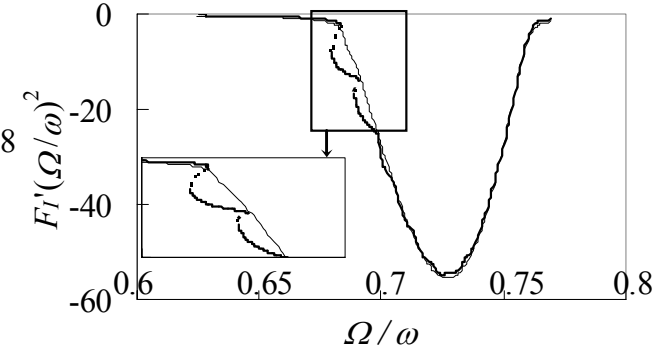

(2) Condition (b) $, R / h_{0}=12.0, X^{\prime}=0.020$

Fig. 5 Fluid force response

(analysis: $a_{0}=0.4$, stable; $a_{0}=0.4$, unstable; ,$a_{0}=1.6$, stable)

On the retrograde wave in condition (a), the fluid force responses with different liquid depths (liquid layer thickness) and with the fixed excitation amplitude of $X=1 \mathrm{~mm}$ are shown in Fig. 6(1), whereas those with different excitation amplitudes and the fixed liquid depth of $h_{0}=20 \mathrm{~mm}$ are shown in Fig. 6(2). These figures show the responses of the total fluid force acting on the entire cylindrical container, $F_{R} L$ and $F_{I} L$, where $L$ is the axial length of the container $(L=49 \mathrm{~mm}$ in condition (a)). Both non-linear $(N=16)$ and linear $(N=1)$ analytical results are shown in the figures. Unstable speed ratio bounds of an elastically supported rotor obtained by Wolf's linear theory ${ }^{(2)}$ are also shown. In the calculation of unstable bounds by Wolf's theory, we assume that the mass of the empty rotor with a support system is much greater than the mass of the fluid and we set the mass ratio as $\rho \pi R^{2} / M=0.05$ (where $M$ is the mass per unit length of the empty rotor). We also assume the whirl frequency is identical to the natural frequency of the empty rotor on the support system, because the frequency of the self-excited whirl is generally close to the natural frequency of the empty rotor and its support system. The imaginary part of the force, $F_{I} L$, has the role of excitation in an asynchronous whirl. In both Figs. 6(1) and 6 (2), the speed regions in which $F_{I} L$ occurs in non-linear analyses are in agreement with unstable bounds 
by Wolf's theory. The experimental results show jump phenomena by the change in the rotating speed ratio $\Omega / \omega$, as is observed in the wave height response (Fig. 3), and $F_{I} L$ in the experiment then shows its peak at the speed ratio upper than the analytical peak. The analytical results show no jump phenomenon. However, focusing on the fluid force at the speed ratio $\Omega / \omega$ at which the imaginary part reaches its peak, we find that both the real and imaginary parts obtained by the non-linear analysis are, on the whole, in good agreement with the experimentally obtained values, although the analysis slightly overestimates the imaginary parts in cases with different liquid depths. Peak values of $F_{R} L$ and $F_{I} L$ of linear analyses $(N=1)$ are much greater than those of non-linear analyses $(N=16)$. This indicates that taking into account nonlinearities of the free surface wave, i.e., non-linear coupling

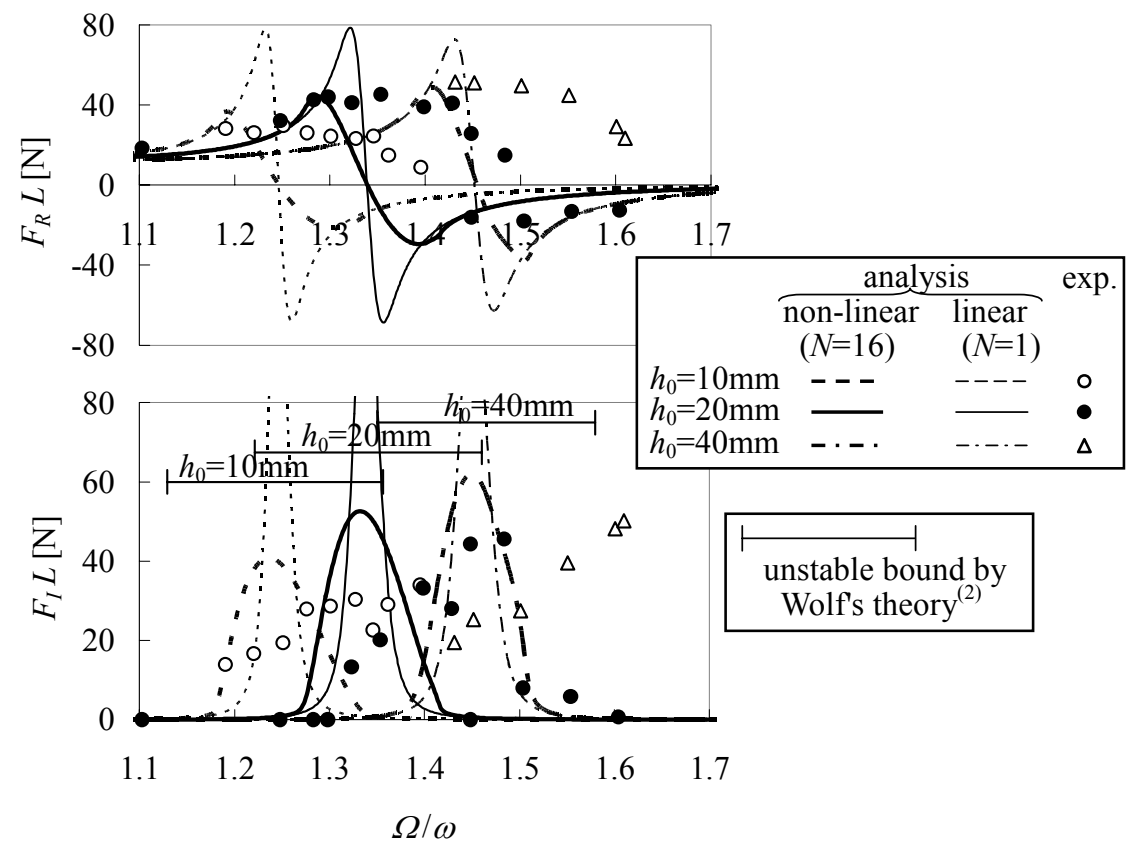

(1) Effect of fluid layer thickness $h_{0}(X=1 \mathrm{~mm})$

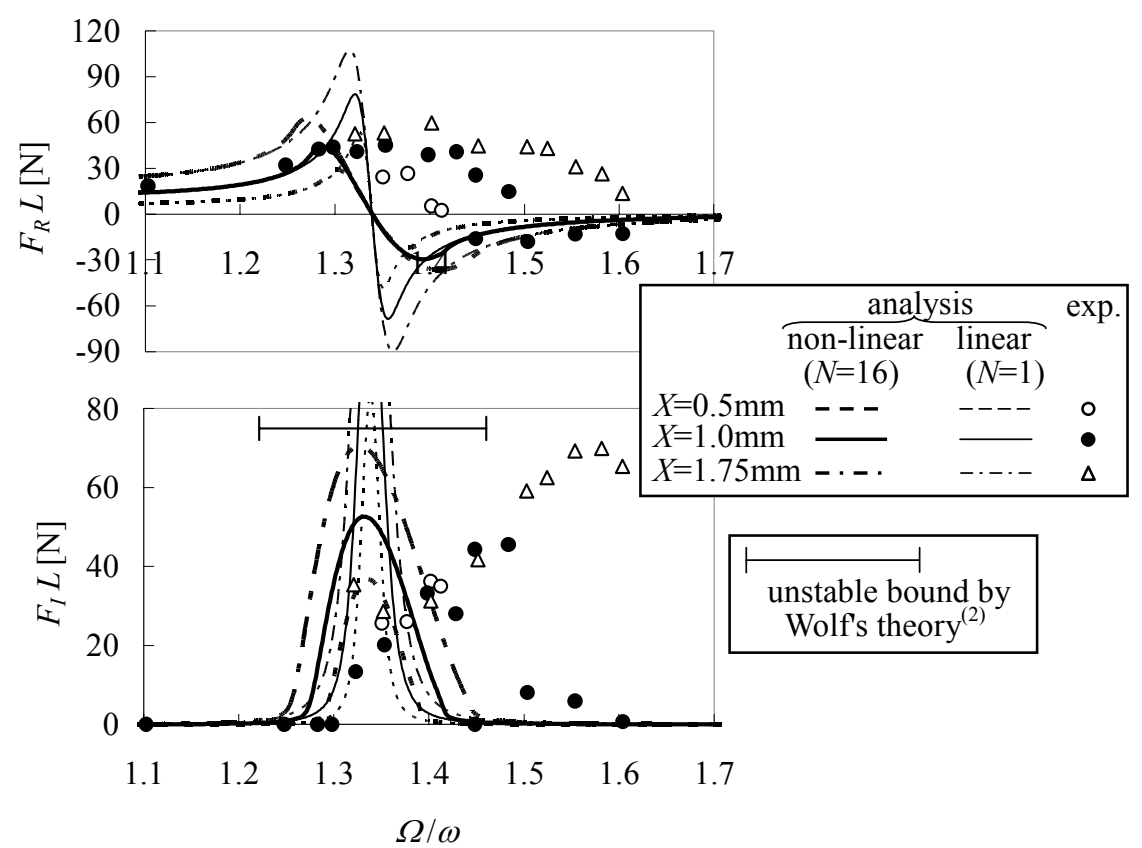

(2) Effect of whirl amplitude $X\left(h_{0}=20 \mathrm{~mm}\right)$

Fig. 6 Fluid force response (effect of fluid layer thickness and whirl amplitude in condition (a), experiment: Inoue et al. (1985), $a_{0}=1.6$ in analysis) 
between modes is necessary to predict the magnitude of the fluid force. The differences in the response curve, particularly the jump phenomenon and the rotating speed ratio at which the imaginary part reaches its peak, between the present analysis and the experiment are believed to be due to the simplified turbulence model of the eddy viscosity. One ground for this is the analytical results by Inoue, Jinnouchi and Araki ${ }^{(8)}$. They have shown, in response curves obtained by the analysis using the theory of hydraulic jump, the rotating speed at which the imaginary part reaches its peak is approximate to that in the experiment. The present model is considered not to be sufficient to precisely predict the actions of a large wave with a bore, but the quantitative agreement on the magnitude of the fluid force is good.

\section{Conclusions}

Nonlinear analyses based on the shallow-water approximation were performed in order to predict nonlinear actions of free surface waves propagating on a spun up liquid film in a hollow rotor undergoing harmonic whirl motions. Analytical results were compared with experimental results reported in previous studies. Although there is a lack of agreement with respect to the rotating speed of the jump phenomenon in the response curves, the analytical and experimental results show that, on the whole, good qualitative and quantitative agreement was observed with respect to the fluid force. Since the whirl is generated by the fluid force, the present theory is expected to provide an accurate prediction of the whirl amplitude. The analyses of the present study revealed that the selection of a suitable eddy viscosity provides an accurate prediction of experimental wave height profiles.

\section{Acknowledgement}

The author would like to thank Mitsui Engineering \& Shipbuilding Co., Ltd. for the opportunity to conduct this research and for engaging in numerous useful discussions.

\section{Appendix Specific expressions of fluid forces}

$$
\left.\begin{array}{c}
F_{1}=\pi \rho R\left\{\frac{1}{2} h_{0} \ddot{C}_{1}+2 R \Omega \dot{D}_{1}+R \Omega^{2} C_{1}+X \omega^{2} h_{0} \cos \sigma t+\frac{R}{h_{0}} \sqrt{\frac{E|\sigma|}{2}}\left(\operatorname{sign}(\sigma) \dot{D}_{1}+\dot{C}_{1}\right)\right\} \\
\left.F_{2}=\pi \rho R\left\{\frac{1}{2} h_{0} \ddot{D}_{1}-2 R \Omega \dot{C}_{1}+R \Omega^{2} D_{1}-X \omega^{2} h_{0} \sin \sigma t+\frac{R}{h_{0}} \sqrt{\frac{E|\sigma|}{2}}\left(-\operatorname{sign}(\sigma) \dot{C}_{1}+\dot{D}_{1}\right)\right\}\right\}, \\
F_{R}=\pi \rho R\left[\left(-\frac{1}{2} h_{0} \sigma^{2}-2 R \Omega \sigma+R \Omega^{2}\right) c_{1}+\left(h_{0} \sigma+2 R \Omega\right) \dot{d}_{1}+X \omega^{2} h_{0}\right. \\
\left.+\frac{R}{h_{0}} \sqrt{\frac{E|\sigma|}{2}}\left\{\operatorname{sign}(\sigma)\left(-\sigma c_{1}+\dot{d}_{1}\right)+\sigma d_{1}+\dot{c}_{1}\right\}\right] \\
F_{I}=\pi \rho R\left[\left(-\frac{1}{2} h_{0} \sigma^{2}-2 R \Omega \sigma+R \Omega^{2}\right) d_{1}-\left(h_{0} \sigma+2 R \Omega\right) \dot{c}_{1}\right. \\
\left.+\frac{R}{h_{0}} \sqrt{\frac{E|\sigma|}{2}}\left\{-\operatorname{sign}(\sigma)\left(\sigma d_{1}+\dot{c}_{1}\right)-\sigma c_{1}+\dot{d}_{1}\right\}\right]
\end{array}\right\}
$$

\section{References}

(1) Miles, J. W., and Troesch, B. A., Surface Oscillations of a Rotating Liquid, Transaction of the ASME, Journal of Applied Mechanics, vol. 28, (1961), pp. 491-496.

(2) Wolf, J. A., Whirl Dynamics of a Rotor Partially Filled with Liquid, Transaction of the 
ASME, Journal of Applied Mechanics, Vol. 35, (1968), pp. 676-682.

(3) Ehrich, F. F., The Influence of Trapped Fluid on High Speed Rotor Vibration, Transaction of the ASME, Journal of Engineering for Industry, Vol. 89, (1967), pp. 806-812.

(4) Saito, S. and Someya, T., Investigation into the Vibration of a Rotating Hollow Shaft Partially Filled with Liquid -1st Report, Numerical Solution of Liquid Force by the Finite Difference Method, Transactions of the Japan Society of Mechanical Engineers, Series C, (in Japanese), Vol. 44, No. 388, (1978), pp. 4115-4122.

(5) Hendricks, S. L. and Morton, J. B., Stability of a Rotor Partially Filled with a Viscous Incompressible Fluid, Transaction of the ASME, Journal of Applied Mechanics, Vol. 46, (1979), pp. 913-918.

(6) Kaneko, S. and Hayama, S., On Free Surface Oscillations of Liquid Partially Filling a Rotating Cylinder - 1st Report, Visualization of Resonant Mode and Analysis by Inviscid Theory, Transactions of the Japan Society of Mechanical Engineers, Series C, (in Japanese), Vol. 49, No. 439, (1983), pp. 370-380.

(7) Berman, A. S., Lundgren, T. S., and Cheng A., Asynchronous Whirl in a Rotating Cylinder Partially Filled with Liquid, Journal of Fluid Mechanics, Vol. 150, (1985), pp. 311-327.

(8) Inoue, J., Jinnouchi, Y. and Araki, Y., Forced Wave Motion of Liquid Partially Filling a High-Speed Rotor, Transaction of the ASME, Journal of Vibration, Acoustics, Stress, and Reliability in Design, Vol. 107, (1985), pp. 446-452.

(9) Colding-Jorgensen, J., Rotor Whirl Measurements on a Long Rotating Cylinder Partially Filled with Liquid, Transaction of the ASME, Journal of Vibration and Acoustics, Vol. 115, (1993), pp. 141-144.

(10) Kasahara, M., Kaneko, S., Oshita, K. and Ishii, H., Experiment of Liquid Motion in a Whirling Ring, Abstracts of Dynamics \& Design Conference, Japan Society of Mechanical Engineers, (in Japanese), (2000), No. 339

(11) Kasahara, M., Kaneko, S. and Ishii, H., Vibration Analysis of Rotors with a Fluid Balancer - 1st Report, Shallow-Water Sloshing Analysis of Liquid in a Whirling Ring, Transactions of the Japan Society of Mechanical Engineers, Series C, (in Japanese), Vol. 66, No. 646, (2000), pp. 1762-1768.

(12) Hutton, R. E., An Investigation of Resonant, Nonlinear, Nonplanar Free Surface Oscillations of a Fluid, NASA Technical Note, D-1870(1963), pp. 1-64.

(13) Miles, J. W., Nonlinear Surface Waves in Closed Basins, Journal of Fluid Mechanics, Vol. 75(1976), pp. 419-448.

(14) Faltinsen, O. M., Rognebakke, O. F., Lukovsky, I. A. and Timokha, A. N, Multidimensional Modal Analysis of Nonlinear Sloshing in a Rectangular Tank with Finite Water Depth, Journal of Fluid Mechanics, Vol. 407(2000), pp. 201-234.

(15) Kimura, N. and Ohashi, H., Nonlinear Sloshing in Containers with Arbitrary Axisymmetric Geometries - 1st Report, Derivation of Governing Equations and the Behavior of Their Solutions, Transactions of the Japan Society of Mechanical Engineers, (in Japanese), Vol. 44, No.385 (1978), pp. 3024-3033.

(16) Sakata, M., Kimura, K. and Utsumi, M., Non-Stationary Response of Non-Linear Liquid Motion in a Cylindrical Tank subjected to Random Base Excitation, Journal of Sound and Vibration, Vol. 94, No.3, (1984), pp. 351-363.

(17) Ikeda, T., and Nakagawa, N., Nonlinear Vibrations of a Structure Caused by Water Sloshing in a Rectangular Tank, Journal of Sound and Vibration, Vol. 201, No.1, (1997), pp. 23-41.

(18) Chester, W., Resonant Oscillations of Water Waves, Proceedings of the Royal Society of London, A306, (1968), pp. 5-22.

(19) Fukasawa, M., Iwase, H., Gotoh, C. and Iida, K., A Numerical Model for Soliton Fission and Wave Breaking of Shallow Water Waves, Abstracts of Proceeding of The School of Engineering of Tokai University, (in Japanese), vol. 41, No. 2, (2001), pp. 93-98. 